\title{
LED-based Photometric Stereo-Imaging Employing Frequency-Division Multiple Access
}

\author{
Johannes Herrnsdorf ${ }^{1}$, Jonathan McKendry ${ }^{1}$, Mark Stonehouse ${ }^{1}$, Laurence Broadbent ${ }^{2}$, Glynn C. Wright ${ }^{2}$, \\ Martin D. Dawson ${ }^{1}$, and Michael J. Strain ${ }^{1}$ \\ ${ }^{1}$ Institute of Photonics, Department of Physics, University of Strathclyde, Glasgow G1 1RD (UK), \\ ${ }^{2}$ Aralia Systems, Bristol Robotics Laboratory, Bristol BS16 1QY (UK), \\ Email: johannes.herrnsdorf@ strath.ac.uk
}

\begin{abstract}
We present a photometric stereo-imaging approach based on illumination with light-emitting diodes (LEDs) from different angles where the LEDs are sinusoidally modulated and do not require synchronization with each other or with the camera.
\end{abstract}

\section{INTRODUCTION}

Among the many techniques developed for 3D imaging, photometric stereo-imaging excels through the simplicity of the hardware required. It can be used stand-alone or in fusion with other 3D imaging techniques for applications such as 3D scanners, face recognition or surveillance applications [1], [2], [3], [4], [5]. In photometric stereo-imaging, the subject is illuminated by different light sources from different angles, and the surface shades created by each light source allow reconstruction of $3 \mathrm{D}$ information.

The vast majority of photometric stereo-imaging experiments used a time-division multiple access scheme (TDMA), where only one light source was switched on at a time allowing the camera to capture the illumination from only this particular source. This approach requires synchronization of all light sources with the camera or at least, if the camera oversamples the illumination, of all light sources with each other. Hernandez et al. [2] reported photometric stereo-imaging with wavelength division multiple access, where three illumination sources were used and emitted red, green and blue light. By this approach, they removed the need for synchronization and achieved a high framerate of 60 fps. However, this approach has significant limitations. It is very difficult to extend the scheme to more than 3 light sources, because with widely available cameras the selection of wavelength channels is limited to red, green and blue. The colored lights may lead to unpleasant visual perception if the system is installed in a public space (e.g. for surveillance purposes) and the 3D reconstruction may not work well for colored subjects.

Here, we report a photometric stereo-imaging technique based on frequency-division multiple access (FDMA), where each light source is modulated sinusoidally. For each camera pixel, a power Fourier transform (PFT) of the time-sequence intensity of that pixel allows identification of the frequency components and thus the contribution of each light source to the intensity recorded by the pixel. FDMA has been used for visible light positioning before [6] but this is the first time to our knowledge that it is applied to 3D imaging. Besides

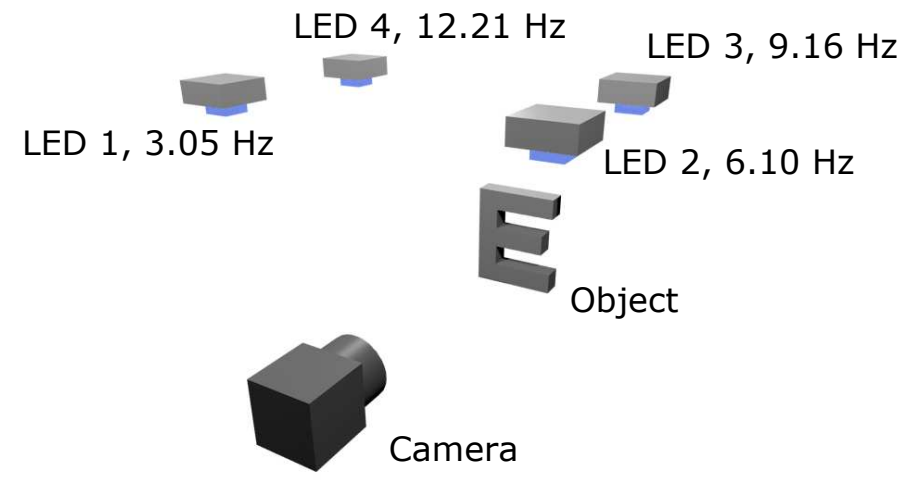

Fig. 1. Schematic of the experimental setup.

removing the need for synchronisation, photometric stereoimaging using FDMA has two more advantages to TDMA. In TDMA the LED duty cycle is $1 / N$ with $N$ being the number of LEDs, whereas for FDMA it is $50 \%$, thus yielding a higher illumination level. FDMA also filters any constant background light in the scene.

\section{EXPERIMENT}

We used four white LEDs (Osram OSTAR Stage LE RTDUW S2W) with a nominal operating current of $0.7 \mathrm{~A}$, $200 \mathrm{~lm}$ luminous flux, and 8.6 MHz optical bandwidth [7]. The placement of the LEDs and camera, shown in Fig. 1, resembled a typical surceillance scenario. The LEDs are placed in the ceiling in positions typical for room lighting and the camera monitors the scene from the side. This layout is motivated by the practical applications of this approach and is different from conventional photometric stereo-imaging setups where the illumination sources and the camera are all placed in one plane perpendicular to the camera axis. The camera captured frames with a resolution of $640 \times 480$ at a rate of $60 \mathrm{fps}$ and the LEDs were intensity modulated using digital outputs from a field-programmable gate array (FPGA). The digital modulation from the FPGA resulted in full on-off modulation of each LED at a rate of $1 \mathrm{Mb} / \mathrm{s}$. Sinusoidal intensity modulation was achieved by transmitting a pseudo-random bit sequence with sinusoidal duty cycle modulation, i.e. the probability of transmitting a '1' was varied sinusoidally. At the frame rate of the camera this resulted in high fidelity sinusoidal received 
a)

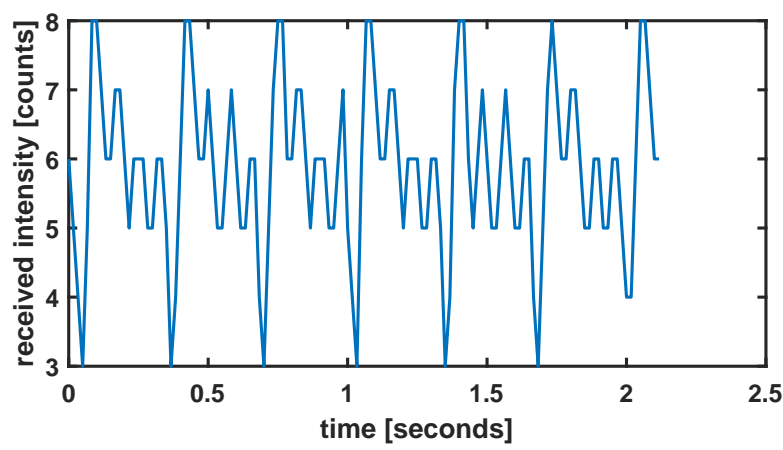

b)

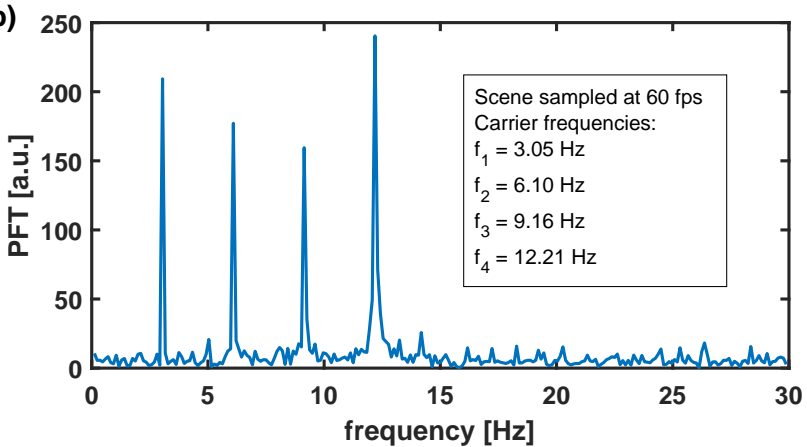

Fig. 2. a) Time sequence received intensity of one representative pixel of the scene and $b$ ) corresponding PFT.

signals at $3.05 \mathrm{~Hz}, 6.10 \mathrm{~Hz}, 9.16 \mathrm{~Hz}$, and $12.21 \mathrm{~Hz}$, which are assigned to the different LEDs as indicated in Fig. 1.

\section{RESULTS}

A representative time sequence of the intensity recorded by one camera pixel is shown in Fig. 2a). It can clearly be seen that the signal is periodical with a period of about $0.33 \mathrm{~s}$, corresponding to the carrier with the lowest frequency. When looking at the PFT of this trace, which is shown in Fig. 2b), four distinct peaks can be made out at the carrier frequencies of the four LEDs.

All pixels of the camera will receive a similar periodical signal, but the intensities of the peaks in the PFT will vary according the the reflectivity and shading of the scene at the position corresponding to the pixel upon illumination from the different LEDs. If we construct an image where the pixel intensity values correspond to the intensity of the PFT at one of the four carrier frequencies then this image will show the scene as if it were illuminated by the corresponding LED only. Such images are shown in the insets of Fig. 3 for all four carrier frequencies. Fig. 3 also shows the surface normal vector that was reconstructed from these images. In this plot, left and right facing orientations lead to red and green appearance, respectively. It can clearly be seen how the orientations of left and right facing sides of the imaged letter "E" are correctly identified.

\section{CONCLUSION}

We have shown how FDMA can be used in photometric stereo-imaging, removing the need for synchronization and

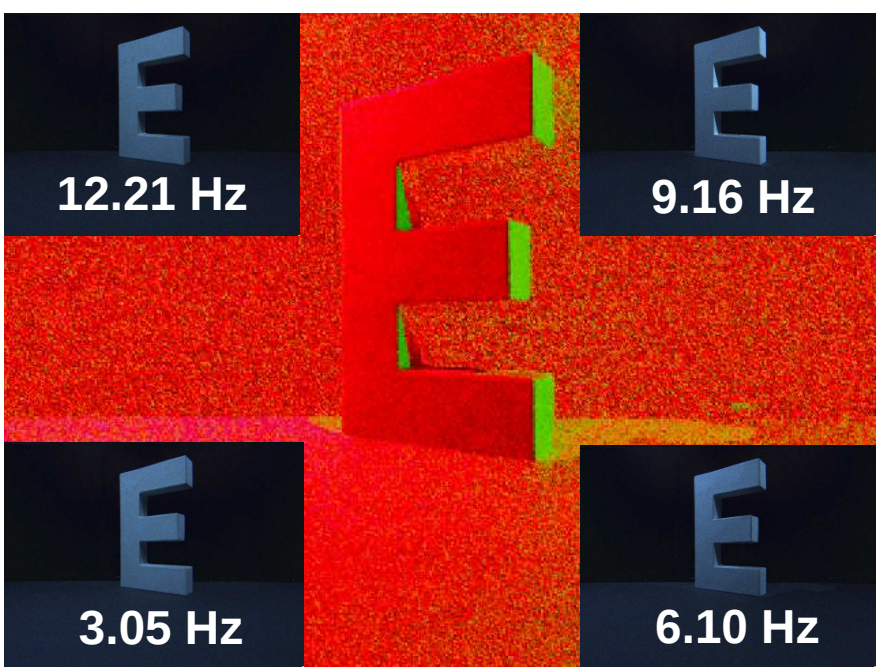

Fig. 3. Reconstructed surface normal vector $\vec{n}$, where $n_{x}$ (pointing up) is encoded in blue, $n_{y}$ (pointing to the right) is encoded in green, and $n_{z}$ (pointing out of the image plane) is encoded in red. Insets: Distribution of the PFT intensity at the carrier frequencies $3.05 \mathrm{~Hz}, 6.10 \mathrm{~Hz}, 9.16 \mathrm{~Hz}$, and $12.21 \mathrm{~Hz}$.

increasing LED duty cycle compared to the conventional TDMA approach. This allows simpler installation of 3D imaging systems. Notably, correct 3D reconstruction was achieved using ceiling-mounted lighting rather than distributing the LEDs in the camera plane. Ongoing work concerns the tradeoffs between sampling time and image quality.

\section{ACKNOWLEDGMENT}

The authors thank the Engineering and Physical Sciences Research Council for funding under Partnership Resource of QuantIC, grant number EP/M01326X/1. Data are available online at http://dx.doi.org/10.15129/30c8cf91-1ebb-479a$9 b 33-2695 c 6248426$.

\section{REFERENCES}

[1] L. Zhang, N. Snavely, B. Curless, and S. M. Seitz, Spacetime Faces: High-Resolution Capture for Modeling and Animation. London: Springer London, 2008, pp. 248-276.

[2] C. Hernandez, G. Vogiatzis, G. J. Brostow, B. Stenger, and R. Cipolla, "Non-rigid photometric stereo with colored lights," in 2007 IEEE 11th International Conference on Computer Vision, Oct 2007, pp. 1-8.

[3] A. F. Martins, M. Bessant, L. Manukyan, and M. C. Milinkovitch, "(ROBBIE)-O-2-3D, a Fast Robotic High-Resolution System for Quantitative Phenotyping of Surface Geometry and Colour-Texture," PLOS $O N E$, vol. 10, no. 6, JUN 32015.

[4] T. Weise, B. Leibe, and L. V. Gool, "Fast 3d scanning with automatic motion compensation," in 2007 IEEE Conference on Computer Vision and Pattern Recognition, June 2007, pp. 1-8.

[5] Y. Zhang, G. M. Gibson, R. Hay, R. W. Bowman, M. J. Padgett, and M. P. Edgar, "A fast 3D reconstruction system with a low-cost camera accessory," SCIENTIFIC REPORTS, vol. 5, JUN 92015.

[6] M. T. Taylor and S. Hranilovic, "Angular diversity approach to indoor positioning using visible light," in 2013 IEEE Globecom Workshops (GC Wkshps), Dec 2013, pp. 1093-1098.

[7] J. Herrnsdorf, L. Broadbent, G. C. Wright, M. D. Dawson, and M. J. Strain, "Video-rate photometric stereo-imaging with general lighting luminaires," in 2017 IEEE Photonics Conference, 2017. 